\title{
LARGE DIFFUSIVITY FINITE-DIMENSIONAL ASYMPTOTIC BEHAVIOUR OF \\ A SEMILINEAR WAVE \\ EQUATION
}

\section{ROBERT WILLIE}

Received 20 December 2002

We study the effects of large diffusivity in all parts of the domain in a linearly damped wave equation subject to standard zero Robin-type boundary conditions. In the linear case, we show in a given sense that the asymptotic behaviour of solutions verifies a second-order ordinary differential equation. In the semilinear case, under suitable dissipative assumptions on the nonlinear term, we prove the existence of a global attractor for fixed diffusion and that the limiting attractor for large diffusion is finite dimensional.

\section{Introduction}

Let $\Omega \subset \mathbb{R}^{N}, N \geq 1$, be an open bounded convex subset with smooth boundary $\partial \Omega=\Gamma$ and consider the following semilinear wave equation:

$$
u_{t t}+\beta u_{t}+L^{\varepsilon} u=f(u), \quad\left(u(0), u_{t}(0)\right)=\left(u_{0}^{\varepsilon}, u_{1}^{\varepsilon}\right) \in X_{\varepsilon}^{1 / 2} \times X_{\varepsilon}^{0},
$$

where $u$ is the unknown, $\beta \geq 1$ is a linear damping term, $\varepsilon>0$ is a given parameter, and $L^{\varepsilon}: H^{1}(\Omega) \mapsto H^{-1}(\Omega)$ denotes the canonic spatial secondorder differential isomorphic operator incorporating the boundary conditions $\left\langle d^{\varepsilon}(x) \nabla u, \vec{n}\right\rangle+b^{\varepsilon}(x) u=0$ on $\Gamma$ with $\vec{n}$ being the external unit normal vector to $\Gamma$. More precisely, we consider the bilinear form $a^{\varepsilon}$ : $H^{1}(\Omega) \times H^{1}(\Omega) \mapsto \mathbb{R}$ given as

$$
a^{\varepsilon}(u, \varphi)=\int_{\Omega} d^{\varepsilon}(x) \nabla u \nabla \varphi+\lambda \int_{\Omega} u \varphi+\int_{\Gamma} b^{\varepsilon} u \varphi
$$


such that, for each $u \in H^{1}(\Omega), L^{\varepsilon} u \in H^{-1}(\Omega)$ is defined by

$$
\left\langle L^{\varepsilon} u, \varphi\right\rangle_{H^{-1}(\Omega), H^{1}(\Omega)}=a^{\varepsilon}(u, \varphi) \quad \forall \varphi \in H^{1}(\Omega) .
$$

In (1.2), $d^{\varepsilon} \in C(\Omega)$ is a strictly positive diffusion coefficient and $b^{\varepsilon} \in$ $L^{q_{0}}(\Gamma)$ with $q_{0} \geq 1$ if $N=1, q_{0}>1$ if $N=2$, and $q_{0} \geq N-1$ if $N \geq 3$ is the boundary potential. Let $\lambda_{0}: \mathbb{R}^{+} \mapsto \mathbb{R}^{+}$be such that $\lambda_{0}(0)=0$ and let $b_{-}^{\varepsilon}$ denote the negative part of the potential $b^{\varepsilon}(x)$. Throughout, we will consider the constant $\lambda \geq \lambda_{0}\left(\left\|b_{-}^{\varepsilon}\right\|_{L^{q_{0}}(\Gamma)}\right)$.

We note that, for simplicity in our exposition, we have chosen the given situation on the boundary. This will allow us to set off messy technical hypotheses in our treatment as nonhomogeneous boundary conditions require certain compatibility assumptions to be verified.

Now we make precise the sense in which we will understand the effect of large diffusion in all parts of the domain $\Omega$ of (1.1). This will be entailed in the hypothesis that

$$
D_{\text {inf }}^{\varepsilon} \stackrel{\text { def }}{=} \inf _{\Omega}\left\{d^{\varepsilon}(x)\right\} \longrightarrow \infty \quad \text { as } \varepsilon \longrightarrow 0 .
$$

Simultaneously with assumption (1.4) and corresponding to physical relevant cases (see $[2,12])$, we suppose, for the boundary potential and initial conditions to problem (1.1), that these are uniformly bounded in norm of the spaces in which they reside for $\varepsilon>0$ and satisfy $L^{1}$ convergence as $\varepsilon \rightarrow 0$.

In the initial paragraph, we have stated that the operator $L^{\varepsilon}$ is a canonic isomorphism. This follows the fact that Lax-Milgram theorem $[1,11]$ is satisfied. Indeed, since other hypotheses to be verified are readily seen, it suffices to note that once (1.4) is assumed, we have coercivity in $H^{1}(\Omega)$, that is,

$$
a^{\varepsilon}(u, \varphi) \geq \beta_{1}^{\varepsilon} \int_{\Omega}|\nabla u|^{2}+\beta_{0} \int_{\Omega}|u|^{2} \geq C\|u\|_{H^{1}(\Omega)}^{2}
$$

where $\beta_{1}^{\varepsilon} \rightarrow \infty$ as $\varepsilon \rightarrow 0, C>0$ is independent of $\varepsilon>0$. Thus, throughout, we will suppose that $\varepsilon>0$ is sufficiently small so as to yield (1.5) always.

To complete the precision of the data in (1.1), we will consider a nonlinear reaction term $f \in C^{1}(\mathbb{R})$ satisfying, for $N \geq 3$, polynomial growth conditions of type

$$
|f(u)-f(v)| \leq C\left(|u|^{p-1}+|v|^{p-1}+1\right)|u-v|, \quad \text { for } 1<p \leq \frac{N+2}{N-2},
$$


while if $N=2$ in (1.6), we assume that $p-1=2$. Alternatively, we will suppose that for all $\eta>0$ there exists $C_{\eta} \geq 0$ such that

$$
|f(u)-f(v)| \leq C_{\eta}\left(e^{\eta|u|^{2}}+e^{\eta|v|^{2}}+1\right)|u-v| .
$$

If $N=1$, no growth conditions are required.

Finally, we remark that the norm of the extended scale of Hilbert space $X_{\varepsilon}^{1 / 2}$ (see $[1,8]$ ) is given by $\|u\|_{X_{\varepsilon}^{1 / 2}}^{2}=a^{\varepsilon}(u, u)$, as usual $X_{\varepsilon}^{0}=L^{2}(\Omega)$. We will employ the notation $\langle\cdot, \cdot\rangle$ to denote the inner product of $X_{\varepsilon}^{0}$. We will also use $f .=(1 /|\cdot|) \int$. to denote the spatial average integral operator for functions defined either in $\Omega$ or on its boundary $\Gamma$. Finally, all generic constants independent of $\varepsilon>0$ will be denoted by $C \geq 0$.

Our aim is to investigate the limiting problem for (1.1) in the given hypotheses under the effect of (1.4) and the extent to which the long-time dynamics are related. It is easy to intuitively guess the explicit expression of the limiting large diffusivity equation for (1.1). However, it is not trivial to make a precise meaning for this limiting process. On a similar subject are the paper by Carvalho [3] and sectional conclusions of the monograph by Hale [6]. Both these references are technically different from the present paper since they prove an inverse situation to the one we have outlined at the beginning of the paragraph.

It is worthwhile noting that the pioneer work of Conway et al. [4] implies a fine exponential decay of solutions to a constant function in space for a system of reaction-diffusion equations subject to zero Neumann boundary conditions and admitting an invariant region when large diffusivity is assumed in all parts of the domain, consequently a finitedimensional asymptotic limiting system of equations.

For a complete review on known results giving the effects of large diffusion in reaction-diffusion equations, we refer the reader to the introductory chapter of Willie [12]. There we have also provided a bibliography of interest in the topic from other natural sciences. From a mathematical point of view, this asymptotic behaviour of solutions to infinite-dimensional problems is of intrinsic interest in itself since finitedimensional problems turn out to be relatively easier.

We now outline the structure of the paper. In Section 2, we will carefully study the convergence as $\varepsilon \rightarrow 0$ under assumption (1.4) of solutions of an associated linear problem with zero damping term and model hypotheses to (1.1). This will give us an insight to the limiting problem for the semilinear case under large diffusivity. In Section 3, we therefore prove in detail local existence and uniqueness of solutions to problem (1.1). In addition, if we suppose a dissipative condition on the nonlinear term in Section 3.1, we obtain global existence and boundedness of solutions to (1.1) in the energy space $X_{\varepsilon}^{1 / 2} \times X_{\varepsilon}^{0}$. In particular, we prove 
the existence of a global compact attractor $\mathscr{A}^{\varepsilon} \subset X_{\varepsilon}^{1 / 2} \times X_{\varepsilon}^{0}$ that captures the long-time asymptotic behaviour of the solutions to (1.1). Lastly, in Section 3.2, we study how varies the family of attractor $\left\{\mathscr{A}^{\varepsilon}\right\}_{\varepsilon} \subset X_{\varepsilon}^{1 / 2}$ $\times X_{\varepsilon}^{0}$ as $\varepsilon \rightarrow 0$ given that assumption (1.4) is satisfied; here we prove the existence of a finite-dimensional asymptotic limiting problem of the solutions.

\section{The linear evolutionary problem}

In this section, we study the convergence of solutions as $\varepsilon \rightarrow 0$ in the following linear evolutionary wave equation:

$$
u_{t t}+L^{\varepsilon} u=f^{\varepsilon}(t), \quad u^{\varepsilon}(0)=u_{0}^{\varepsilon} \in X_{\varepsilon}^{1 / 2}, \quad u_{t}^{\varepsilon}(0)=u_{1}^{\varepsilon} \in X_{\varepsilon}^{0},
$$

where $f^{\varepsilon} \in L^{1}\left(0, T, L^{2}(\Omega)\right)$ is well behaved for all $\varepsilon>0$ and has spatial average weakly converging in $L^{1}(0, T)$ as $\varepsilon \rightarrow 0$. Note that in (2.1), for simplicity, we have assumed that $\beta=0$. Now, regarding its solvability, we have the following theorem.

THEOREM 2.1. The evolutionary problem (2.1) has a unique weak solution $\left(u^{\varepsilon}, u_{t}^{\varepsilon}\right) \in Y_{T}=C\left([0, T], X_{\varepsilon}^{1 / 2} \times X_{\varepsilon}^{0}\right)$ and the energy identity

$$
\frac{1}{2} E^{\varepsilon}(t)-\frac{1}{2} E^{\varepsilon}(s)=\int_{s}^{t}\left\langle f^{\varepsilon}, u_{t}^{\varepsilon}\right\rangle d \sigma, \quad \forall t \geq s \geq 0,
$$

where $E^{\varepsilon}(t)=\left\|\left(u^{\varepsilon}, u_{t}^{\varepsilon}\right)\right\|_{X_{\varepsilon}^{1 / 2} \times X_{\varepsilon}^{0}}^{2}$, holds.

Proof. The proof of the first part of the theorem is standard and can be found in $[9,11]$. It remains only to show that the energy identity $(2.2)$ holds, but this is obtained via a density argument similar to the one used below in the semilinear case.

We comment that the proof given in [11] is simple and makes use of the standard Galerkin technique. This technique consists formally in deriving an energy inequality from (2.2), which yields among other things an a priori estimate for the solution, expressing as well a continuous dependence relation of this with respect to the data of the problem. Then use an approximation scheme and uniform energy estimate of the approximating sequence of solutions to associated finite-dimensional problems, for which existence is known a priori, to obtain a solution to (2.1) as a weak limit. Since the weak solution obtained by this process depends on the choice of the approximation scheme, the energy estimate yields uniqueness only if more regularity on the data is assumed. 
Thus, to circumvent this difficulty, Renardy and Rogers [11] derive an energy equation for time-integrated quantities which, together with the well-known Gronwall lemma, conclude the desired uniqueness.

We now turn back to our main goal of the section and we have the following asymptotic behaviour of solutions to (2.1).

THeorem 2.2. Consider the second-order ordinary differential equation

$$
\begin{aligned}
& \ddot{u}_{\Omega}+\left(\frac{|\Gamma|}{|\Omega|} f_{\Gamma} b+\lambda\right) u_{\Omega}=h_{\Omega}(t), \\
& \left(u_{\Omega}(0), \dot{u}_{\Omega}(0)\right)=\left(u_{\Omega}^{0}, u_{\Omega}^{1}\right) \in \mathbb{R}^{2},
\end{aligned}
$$

where $h_{\Omega}(t)=f_{\Omega} f d x$, and denote $Q_{T}=\Omega \times(0, T)$. Then the weak solutions to (2.1) satisfy, as $\varepsilon \rightarrow 0$,

$$
u^{\varepsilon}(t) \longrightarrow u_{\Omega}(t), \quad \lim _{\varepsilon \rightarrow 0} \int_{Q_{T}} d^{\varepsilon}(x)\left|\nabla u^{\varepsilon}\right|^{2}=0,
$$

where the first convergence is strong in $L^{2}\left(0, T, X_{\varepsilon}^{1 / 2}\right)$. If there is in addition a strong convergence of the data as $\varepsilon \rightarrow 0$, then

$$
\left(u^{\varepsilon}, u_{t}^{\varepsilon}\right) \longrightarrow\left(u_{\Omega}, \dot{u}_{\Omega}\right) \text { strongly in } \Upsilon_{T} \text { as } \varepsilon \longrightarrow 0 .
$$

Proof. Consider the energy identity (2.2) for $s=0$, that is,

$$
\frac{1}{2} E^{\varepsilon}(t)=\frac{1}{2} E^{\varepsilon}(0)+\int_{0}^{t}\left\langle f^{\varepsilon}, u_{t}^{\varepsilon}\right\rangle d \sigma \quad \forall t \geq 0 .
$$

Then, applying Hölder's inequality followed by Young's inequality of the form

$$
a b \leq \eta a^{2}+\frac{1}{\eta} b^{2}, \quad a, b \geq 0, \eta=\frac{1}{4}
$$

in the last term of the right-hand side, we obtain

$$
E^{\varepsilon}(t) \leq 16\left(E^{\varepsilon}(0)+\left\|f^{\varepsilon}\right\|_{L^{1}\left(0, T, L^{2}(\Omega)\right)}^{2}\right) .
$$

Since, by hypothesis, the right-hand side of this last expression is uniformly bounded in $\varepsilon>0$, we have that $\left(\mathcal{u}^{\varepsilon}, u_{t}^{\varepsilon}\right)$ is bounded in norm of $L^{\infty}\left(0, T, X_{\varepsilon}^{1 / 2} \times X_{\varepsilon}^{0}\right)$ for all $\varepsilon>0$. Consequently, passing to subsequences if 
414 Finite-dimensional asymptotic behaviour

necessary, we conclude

$$
\left(u^{\varepsilon}, u_{t}^{\varepsilon}\right) \longrightarrow\left(v, v_{t}\right) \text { weak* in } L^{\infty}\left(0, T, X_{\varepsilon}^{1 / 2} \times X_{\varepsilon}^{0}\right)
$$

as $\varepsilon \rightarrow 0$; and since (1.4) is satisfied, we also have for all $t \geq 0$ that

$$
\lim _{\varepsilon \rightarrow 0} \int_{\Omega}\left|\nabla u^{\varepsilon}\right|^{2}=0
$$

Thus, using the lower semicontinuity of the $H^{1}(\Omega)$ norm, we deduce that the limit $v$ is constant in $\Omega$ for all $t \in(0, T)$. In particular, the strong compactness in $X_{\varepsilon}^{0}$ for all $t \in(0, T)$ and (2.10) implies $u^{\varepsilon}(t) \rightarrow v(t)$ strongly in $L^{2}\left(0, T, X_{\varepsilon}^{1 / 2}\right)$ as $\varepsilon \rightarrow 0$. On the other hand, the Poincaré inequality yields

$$
\left\|u^{\varepsilon}-\bar{u}^{\varepsilon}\right\|_{L^{2}\left(Q_{T}\right)} \leq C\left\|\nabla u^{\varepsilon}\right\|_{L^{2}\left(Q_{T}\right)} \longrightarrow 0,
$$

as $\varepsilon \rightarrow 0$, where $\bar{u}^{\varepsilon}=f_{\Omega} u^{\varepsilon}$. Hence, the standard Sobolev inclusions $H^{1}(\Omega)$ $\hookrightarrow L^{\prime}(\Gamma)$ with $q_{0}^{\prime} \geq 1$ satisfying $1 / q_{0}^{\prime}+2 / q_{0}=1$ for $q_{0} \geq 1$, as given in Section 1 and (2.10), imply

$$
\lim _{\varepsilon \rightarrow 0}\left\|u^{\varepsilon}-\bar{u}^{\varepsilon}\right\|_{L^{2}\left(0, T, L^{\left.q_{0}^{\prime}(\Gamma)\right)}\right.}=0 .
$$

Now let $\psi(t)(1 /|\Omega|) \chi_{\Omega}(x)$ with $\psi \in C^{\infty}[0, T]$ satisfying $\psi(T)=0$ and $\chi_{\Omega}$, the characteristic function of the domain $\Omega$, be a test function in (2.1), and integrate by parts to obtain

$$
\begin{gathered}
-\int_{0}^{T} \dot{\psi} f_{\Omega} u_{t}^{\varepsilon}+\int_{0}^{T} \psi\left(\lambda f_{\Omega} u^{\varepsilon}+\frac{1}{|\Omega|} \int_{\Gamma} b^{\varepsilon} u^{\varepsilon}\right) \\
=\psi(0) f_{\Omega} u_{1}^{\varepsilon}+\int_{0}^{T} \psi f_{\Omega} f^{\varepsilon} .
\end{gathered}
$$

Before passing to the limit as $\varepsilon \rightarrow 0$ in (2.13), we set $\Sigma_{T}=\Gamma \times(0, T)$ and observe that

$$
\left|\int_{\Sigma_{T}} b^{\varepsilon}\left(u^{\varepsilon}-\bar{u}^{\varepsilon}\right)\right| \leq C \int_{0}^{T}\left\|u^{\varepsilon}-\bar{u}^{\varepsilon}\right\|_{L^{q_{0}^{\prime}(\Gamma)}} \longrightarrow 0 .
$$

Therefore, as $\varepsilon \rightarrow 0$, we get

$$
-\int_{0}^{T} v_{t} \dot{\psi}+\int_{0}^{T}\left(\frac{|\Gamma|}{|\Omega|} f_{\Gamma} b+\lambda\right) v \psi=v_{1} \psi(0)+\int_{0}^{T}\left(f_{\Omega} f\right) \psi,
$$


and it follows in distributional sense that

$$
\ddot{v}+\left(\frac{|\Gamma|}{|\Omega|} f_{\Gamma} b+\lambda\right) v=\int_{\Omega} f \quad \text { on }(0, T)
$$

Moreover, multiplying (2.16) by $\psi \in C^{\infty}[0, T]$, verifying $\psi(T)=0$, integrating by parts the first term and comparing with (2.15), we conclude that $\dot{v}(0)=v_{1}$.

Now returning to (2.13) and repeating the above limiting process for the identity following a second integration by parts of the first term so that the second time derivative is passed onto $\dot{\psi}$ using the hypotheses on the initial-data condition $u^{\varepsilon}(0)=u_{0}^{\varepsilon}$, we find that $v(0)=v_{0}$, and, by uniqueness of the limit, we must have $\left(v, v_{t}\right)=\left(u_{\Omega}, \dot{u}_{\Omega}\right)$.

To prove the last assertion, take $\boldsymbol{u}^{\varepsilon}$ as a test function in (2.1) and integrate in time to find

$$
\int_{Q_{T}} u_{t t}^{\varepsilon} u^{\varepsilon} d x d t+\int_{0}^{T} a^{\varepsilon}\left(u^{\varepsilon}, u^{\varepsilon}\right) d t=\int_{Q_{T}} f^{\varepsilon} u^{\varepsilon} d x d t
$$

It is easy to see that we can pass to the limit as $\varepsilon \rightarrow 0$ and, since (2.16) is the limit in distributions of (2.1), this yields

$$
\int_{0}^{T} v_{t t} v+\left(\frac{|\Gamma|}{|\Omega|} f_{\Gamma} b+\lambda\right)|v|^{2}+\lim _{\varepsilon \rightarrow 0} \int_{Q_{T}} d^{\varepsilon}(x)\left|\nabla u^{\varepsilon}\right|^{2}=\int_{0}^{T} v f_{\Omega} f .
$$

Now multiplying (2.16) by $v$, integrating in time, and comparing with the above last expression give

$$
\lim _{\varepsilon \rightarrow 0} \int_{Q_{T}} d^{\varepsilon}(x)\left|\nabla u^{\varepsilon}\right|^{2}=0
$$

and the first assertion is proved.

To conclude the proof of the theorem, we find the equation defined by $\varphi^{\varepsilon}=u^{\varepsilon}-u_{\Omega}$ and use the energy inequality (2.8) to obtain

$$
\begin{aligned}
& \left\|\left(\varphi^{\varepsilon}, \varphi_{t}^{\varepsilon}\right)\right\|_{X_{\varepsilon}^{1 / 2} \times X_{\varepsilon}^{0}}^{2} \\
& \quad \leq C\left(\left\|\left(\varphi_{0}^{\varepsilon}, \varphi_{1}^{\varepsilon}\right)\right\|_{X_{\varepsilon}^{1 / 2} \times X_{\varepsilon}^{0}}^{2}+\left\|f^{\varepsilon}(t)-h_{\Omega}(t)\right\|_{L^{1}\left(0, T, L^{2}(\Omega)\right)}^{2}\right) \longrightarrow 0
\end{aligned}
$$

as $\varepsilon \rightarrow 0$, from which the result follows and the proof of the theorem is complete. 
416 Finite-dimensional asymptotic behaviour

Remark 2.3. We can improve on the second convergence in (2.4), but we need, in addition to the given hypotheses, to assume that

$$
\lim _{\varepsilon \rightarrow 0} \int_{\Omega} d^{\varepsilon}(x)\left|\nabla u_{0}^{\varepsilon}\right|^{2}=0
$$

with which, using the energy identity (2.2) and (2.9) for passing to the limit as $\varepsilon \rightarrow 0$, then comparing the result with that of multiplying in (2.3) by $\dot{u}_{\Omega}$ and integrating in time, it follows that

$$
\limsup _{\varepsilon \rightarrow 0} \int_{\Omega} d^{\varepsilon}(x)\left|\nabla u^{\varepsilon}\right|^{2}=0 .
$$

We note that (2.21) is not a restrictive condition since solving for the asymptotic behaviour in question in the elliptic case is natural (see [2]).

\section{The semilinear evolutionary problem}

We are now in a position to study the semilinear problem (1.1). Throughout this section, we will concentrate only on the case $N \geq 3$ and we remark that the argument in the remaining cases is easily adaptable with minor modifications. Thus, to initiate our study, we introduce the following concept of weak solution to the problem.

Definition 3.1. Let $d^{\varepsilon}, b^{\varepsilon} \in C^{1}$. The pair $\left(u^{\varepsilon}, u_{t}^{\varepsilon}\right) \in Y_{T}$ is a weak solution to problem (1.1) if there exists a sequence of regular data $\left(u_{0}^{n}, u_{1}^{n}\right), n=$ $1,2, \ldots$, such that

$$
\left(u_{0}^{n}, u_{1}^{n}\right) \longrightarrow\left(u_{0}^{\varepsilon}, u_{1}^{\varepsilon}\right) \in X_{\varepsilon}^{1 / 2} \times X_{\varepsilon}^{0}, \quad\left(u^{n}, u_{t}^{n}\right) \longrightarrow\left(u^{\varepsilon}, u_{t}^{\varepsilon}\right) \in Y_{T}, \quad \text { as } n \longrightarrow \infty,
$$

where $\left(u^{n}, u_{t}^{n}\right), n=1,2, \ldots$, is a unique sequence of strong solutions to (1.1) corresponding to the above regular initial data.

With respect to the solvability of (1.1), we state the following theorem.

THEOREM 3.2. The semilinear wave evolutionary problem (1.1) has a unique solution $\left(\mathcal{u}^{\varepsilon}, u_{t}^{\varepsilon}\right) \in Y_{T}$ and the energy identity

$$
\frac{1}{2} E^{\varepsilon}(t)-\frac{1}{2} E^{\varepsilon}(s)=\int_{s}^{t}\left\langle f(u), u_{t}\right\rangle d \sigma, \quad \forall t \geq s \geq 0,
$$

holds, where $E^{\varepsilon}(t)=\left\|\left(u, u_{t}\right)\right\|_{X_{\varepsilon}^{1 / 2} \times X_{\varepsilon}^{0}}^{2}+\beta \int_{s}^{t}\left|u_{t}\right|^{2}$. 
Proof. Assume that (3.2) holds and define a nonlinear mapping $\mathcal{F}: Y_{T} \mapsto$ $Y_{T}$ such that if $\left(u, u_{t}\right) \in Y_{T}$, then $\left(v, v_{t}\right)=\mathcal{F}\left(u, u_{t}\right)$ solves the problem

$$
v_{t t}+\beta v_{t}+L^{\varepsilon} v=f(u), \quad v^{\varepsilon}(0)=v_{0}^{\varepsilon} \in X_{\varepsilon}^{1 / 2}, \quad v_{t}^{\varepsilon}(0)=v_{1}^{\varepsilon} \in X_{\varepsilon}^{0} .
$$

Next, fix $\rho>0$ and consider the bounded subset of $Y_{T}$

$$
U=\left\{\left(\varphi, \varphi_{t}\right) \in Y_{T}: \sup _{0 \leq t \leq T}\left\|\left(\varphi, \varphi_{t}\right)\right\|_{X_{\varepsilon}^{1 / 2} \times X_{\varepsilon}^{0}} \leq \rho\right\} .
$$

If we set $\left\|\left(v_{0}, v_{1}\right)\right\|_{X_{\varepsilon}^{1 / 2} \times X_{\varepsilon}^{0}} \leq \rho / 4$, then multiplying by $v_{t}$ in (3.3), we find

$$
\begin{aligned}
& \frac{1}{2}\left\|\left(v, v_{t}\right)\right\|_{X_{\varepsilon}^{1 / 2} \times X_{\varepsilon}^{0}}^{2}+\beta \int_{0}^{t} \int_{\Omega}\left|v_{t}\right|^{2} d x d \sigma \\
& \quad \leq \frac{1}{2}\left\|\left(v_{0}, v_{1}\right)\right\|_{X_{\varepsilon}^{1 / 2} \times X_{\varepsilon}^{0}}^{2}+\int_{0}^{t}\left|\left\langle f(u), v_{t}\right\rangle\right| d \sigma .
\end{aligned}
$$

Since $\beta \int_{0}^{t} \int_{\Omega}\left|v_{t}\right|^{2} \geq 0$, it follows, using the growth conditions on the nonlinear term, that

$$
\frac{1}{2}\left\|\left(v, v_{t}\right)\right\|_{X_{\varepsilon}^{1 / 2} \times X_{\varepsilon}^{0}}^{2} \leq \frac{\rho}{8}+C T|\Omega|^{1 / 2}\left(\rho^{p}+1\right) \sup _{0 \leq t \leq T}\left\|v_{t}\right\|_{X_{\varepsilon}^{0}},
$$

from which the Young's inequality (2.7) implies

$$
\left\|\left(v, v_{t}\right)\right\|_{X_{\varepsilon}^{1 / 2} \times X_{\varepsilon}^{0}}^{2} \leq \frac{\rho}{2}+4\left(C T|\Omega|^{1 / 2}\left(\rho^{p}+1\right)\right)^{2} .
$$

Consequently, if $\rho \gg 1$ is sufficiently large and we choose

$$
T \leq\left(2 C|\Omega|^{1 / 2}\left(\rho^{p}+1\right)\right)^{-1}
$$

yielding $\left\|\left(v, v_{t}\right)\right\|_{X_{\varepsilon}^{1 / 2} \times X_{\varepsilon}^{0}} \leq \rho$, then $\left(v, v_{t}\right) \in U$ and $\mathcal{F}$ maps $U$ onto itself.

In continuation, notice that, for $\left(u, u_{t}\right),\left(w, w_{t}\right) \in U$, if we set $\left(v, v_{t}\right)=$ $\mathcal{F}\left(u, u_{t}\right)$ and $\left(\psi, \psi_{t}\right)=\mathcal{F}\left(w, w_{t}\right)$ so that $\left(\varphi, \varphi_{t}\right)=\left(v-\psi, v_{t}-\psi_{t}\right)$ solves

$$
\varphi_{t t}+\beta \varphi_{t}+L^{\varepsilon} \varphi=f(u)-f(w),
$$

and taking the inner product with $\varphi_{t}$, we have

$$
\begin{aligned}
\left\|\left(\varphi, \varphi_{t}\right)\right\|_{X_{\varepsilon}^{1 / 2} \times X_{\varepsilon}^{0}} & \leq \int_{0}^{T}\left|\left\langle f(u)-f(w), \varphi_{t}\right\rangle\right| d \sigma \\
& \leq 3 C T \rho^{p} \sup _{0 \leq t \leq T}\left\|\left(\varphi, \varphi_{t}\right)\right\|_{X_{\varepsilon}^{1 / 2} \times X_{\varepsilon}^{0}}
\end{aligned}
$$


where again we have used the fact that $\beta \int_{0}^{t} \int_{\Omega}\left|v_{t}\right|^{2} d x d \sigma \geq 0$. Thus, for $\rho \gg 1$ sufficiently large, if we choose $T \leq\left(6 C \rho^{p}\right)^{-1}$, we obtain that $\mathcal{F}$ is a strict contractive mapping and, thanks to the Banach fixed-point theorem, there exists a unique solution $\left(u, u_{t}\right)=\mathcal{F}\left(u, u_{t}\right)$ that solves (1.1).

Now assume that $d^{\varepsilon}, b^{\varepsilon} \in C^{1}$ and, for $n=1,2, \ldots$, let

$$
v^{n} \in C\left((0, T), X_{\varepsilon}^{1}\right) \cap C^{1}\left((0, T), X_{\varepsilon}^{1 / 2}\right) \cap C^{2}\left((0, T), X_{\varepsilon}^{0}\right)
$$

be a regular sequence of solutions to (3.3) with $v_{0}^{n} \rightarrow v_{0} \in X_{\varepsilon}^{1 / 2}, v_{1}^{n} \rightarrow v_{1} \in$ $X_{\varepsilon}^{0}$. Then $\left(v^{n}, v_{t}^{n}\right)$ is Cauchy in $C\left([0, T], X_{\varepsilon}^{1 / 2} \times X_{\varepsilon}^{0}\right)$ and the limit $\left(v, v_{t}\right)$ solves (3.3) in the sense given by

$$
\frac{d}{d t}\left\langle v_{t}, \varphi\right\rangle+\beta\left\langle v_{t}, \varphi\right\rangle+\left\langle L^{\varepsilon} v, \varphi\right\rangle=\langle f(u), \varphi\rangle \text { on }[0, T] \text { a.e. }
$$

Thus, $\left(v, v_{t}\right)=\mathscr{F}\left(u, u_{t}\right)$ is a weak solution to (1.1) and, taking $\varphi=v_{t}$ integrating in time for $t \geq s \geq 0$, we conclude that (3.2) holds, with which the proof is complete.

Remark 3.3. Note that, under sufficient regularity assumptions on the data of problem (1.1), it is usual to prove the well-posedness via abstract semigroup methods. Often in this case one reads the evolutionary problem in the form

$$
\partial_{t} U+\mathscr{A}^{\varepsilon} U=F(U), \quad U_{0}=\left(u_{0}^{\varepsilon}, u_{1}^{\varepsilon}\right)^{\top},
$$

where

$$
\mathscr{A}^{\varepsilon}=\left(\begin{array}{cc}
0 & -I \\
L^{\varepsilon} & \beta
\end{array}\right), \quad U=\left(u, u_{t}\right)^{\top}, \quad F(U)=(0, f(u))^{\top} .
$$

Further, the nonlinear mapping in the proof of Theorem 3.2 is given by the variation of the constants formula

$$
\mathcal{F}(U)(t)=e^{-\mathscr{A}^{\varepsilon} t} U_{0}+\int_{0}^{t} e^{-\mathscr{A}^{\varepsilon}(t-s)} F(U(s)) d s
$$

in appropriate functional spaces.

\subsection{Global existence and boundedness of solutions}

We now study the global existence and boundedness of solutions to (1.1). Here our arguments use the same technique as that found in [7]. 
We would like to point out that we were not able to extend the method to cover the case of zero damping, that is, $\beta=0$ in (1.1).

In what follows, we assume that the dissipative condition

$$
\limsup _{|u| \rightarrow \infty} \frac{f(u)}{u}<0
$$

holds, with which we state the following theorem.

THEOREM 3.4. Consider the evolutionary equation (1.1) and suppose in (1.6) that $p<1+2 / N$. Then there exists a nonnegative constant $C \geq 0$ such that if $\left\|\left(u_{0}^{\varepsilon}, u_{1}^{\varepsilon}\right)\right\|_{X_{\varepsilon}^{1 / 2} \times X_{\varepsilon}^{0}} \leq \rho$ for some $\rho>0$, then $\left\|\left(u^{\varepsilon}(t), u_{t}^{\varepsilon}(t)\right)\right\|_{X_{\varepsilon}^{1 / 2} \times X_{\varepsilon}^{0}} \leq C$ for all $t \geq t_{0}(\rho)$. In other words, the semilinear problem is bounded dissipative and also

$$
\int_{0}^{\infty}\left\|u_{t}^{\varepsilon}\right\|_{L^{2}(\Omega)}^{2} \leq C
$$

Proof. Consider, for all $t \geq 0$, the functional

$$
J^{\varepsilon}\left(\psi, \psi_{t}\right)=\frac{1}{2}\left\|\left(\psi, \psi_{t}\right)\right\|_{X_{\varepsilon}^{1 / 2} \times X_{\varepsilon}^{0}}^{2}+\frac{b}{2} \int_{\Omega} \psi \psi_{t}-\int_{\Omega} F(\psi), \quad \text { for } 0<b<1,
$$

where $F(\psi)=\int_{0}^{\psi} f(s) d s$. Finding the time derivative of $J^{\varepsilon}$ for $\left(\psi, \psi_{t}\right)=$ $\left(u^{\varepsilon}, u_{t}^{\varepsilon}\right)$ a solution to $(1.1)$, we have

$$
\begin{aligned}
\frac{d J^{\varepsilon}\left(u^{\varepsilon}, u_{t}^{\varepsilon}\right)}{d t}= & \frac{d}{2 d t}\left\|\left(u^{\varepsilon}, u_{t}^{\varepsilon}\right)\right\|_{X_{\varepsilon}^{1 / 2} \times X_{\varepsilon}^{0}}^{2}+\frac{b}{2} \int_{\Omega}\left|u_{t}^{\varepsilon}\right|^{2} \\
& +\frac{b}{2} \int_{\Omega} u^{\varepsilon} u_{t t}^{\varepsilon}-\int_{\Omega} f\left(u^{\varepsilon}\right) u_{t}^{\varepsilon} \\
= & -\beta \int_{\Omega}\left|u_{t}^{\varepsilon}\right|^{2}+\frac{b}{2} \int_{\Omega}\left|u_{t}^{\varepsilon}\right|^{2}-\frac{b \beta}{2} \int_{\Omega} u^{\varepsilon} u_{t}^{\varepsilon} \\
& -\frac{b}{2}\left\|u^{\varepsilon}\right\|_{X_{\varepsilon}^{1 / 2}}^{2}+\frac{b}{2} \int_{\Omega} f\left(u^{\varepsilon}\right) u^{\varepsilon} \\
\leq & -\frac{\beta}{2} \int_{\Omega}\left|u_{t}^{\varepsilon}\right|^{2}-\frac{b}{2} \int_{\Omega} u^{\varepsilon} u_{t}^{\varepsilon}-\frac{b}{2}\left\|u^{\varepsilon}\right\|_{X_{\varepsilon}^{1 / 2}}^{2}+\frac{b}{2} \int_{\Omega} f\left(u^{\varepsilon}\right) u^{\varepsilon} \\
= & -b\left(\frac{1}{2} \int_{\Omega}\left|u_{t}^{\varepsilon}\right|^{2}+\frac{1}{2} \int_{\Omega} u^{\varepsilon} u_{t}^{\varepsilon}+\frac{1}{2}\left\|u^{\varepsilon}\right\|_{X_{\varepsilon}^{1 / 2}}^{2}\right) \\
& +\frac{b}{2} \int_{\Omega} f\left(u^{\varepsilon}\right) u^{\varepsilon},
\end{aligned}
$$


for any $t \geq 0$, after noticing the result of multiplying by $u_{t}^{\varepsilon}$ in (1.1) and, in the third term of the first line, substituting the expression by its equivalent following a multiplication in (1.1) by $u^{\varepsilon}$, in both cases taking the integral by parts on $\Omega$.

Proceed to observe that [7, Lemma 2.1] implies

$$
\frac{1}{2} \int_{\Omega}\left|u_{t}^{\varepsilon}\right|^{2}+\frac{1}{2} \int_{\Omega} u^{\varepsilon} u_{t}^{\varepsilon}+\frac{1}{2}\left\|u^{\varepsilon}\right\|_{X_{\varepsilon}^{1 / 2}}^{2} \geq \frac{1}{4} \int_{\Omega}\left|u_{t}^{\varepsilon}\right|^{2}+\frac{1}{4}\left\|u^{\varepsilon}\right\|_{X_{\varepsilon}^{1 / 2}}^{2}
$$

Hence, we have in (3.19), for $t \geq 0$, that

$$
\frac{d J^{\varepsilon}\left(u^{\varepsilon}, u_{t}^{\varepsilon}\right)}{d t} \leq-\frac{b}{4}\left(\int_{\Omega}\left|u_{t}^{\varepsilon}\right|^{2}+\left\|u^{\varepsilon}\right\|_{X_{\varepsilon}^{1 / 2}}^{2}\right)+\frac{b}{2} \int_{\Omega} f\left(u^{\varepsilon}\right) u^{\varepsilon} .
$$

Thanks to the dissipative hypothesis (3.16), we have for all $\eta>0$ that there exists $C_{\eta} \geq 0$ such that

$$
f(\varphi) \varphi \leq \eta \varphi^{2}+C_{\eta}, \quad \forall \varphi \in \mathbb{R}
$$

Therefore, with $\eta=b / 8$, we have

$$
\frac{d J^{\varepsilon}\left(u^{\varepsilon}, u_{t}^{\varepsilon}\right)}{d t} \leq-\frac{b}{8}\left\|\left(u^{\varepsilon}, u_{t}^{\varepsilon}\right)\right\|_{X_{\varepsilon}^{1 / 2} \times X_{\varepsilon}^{0}}^{2}+C \quad \forall t \geq 0
$$

On the other hand, following the same assumption leading to (3.22), we notice that

$$
F(\varphi) \leq \eta \varphi^{2}+C_{\eta}, \quad \forall \varphi \in \mathbb{R} .
$$

Hence,

$$
\begin{aligned}
J^{\varepsilon}\left(u^{\varepsilon}, u_{t}^{\varepsilon}\right) \geq & \frac{1}{2}\left\|\left(u^{\varepsilon}, u_{t}^{\varepsilon}\right)\right\|_{X_{\varepsilon}^{1 / 2} \times X_{\varepsilon}^{0}}^{2} \\
& -\frac{b}{2}\left\|u^{\varepsilon}\right\|_{L^{2}(\Omega)}\left\|u_{t}^{\varepsilon}\right\|_{L^{2}(\Omega)}-\frac{1}{8} \int_{\Omega}\left|u^{\varepsilon}\right|^{2}-C \\
\geq & \frac{1}{8}\left\|\left(u^{\varepsilon}, u_{t}^{\varepsilon}\right)\right\|_{X_{\varepsilon}^{1 / 2} \times X_{\varepsilon}^{0}}^{2}-C
\end{aligned}
$$


for any $t \geq 0$. Analogously, we can estimate the functional $J^{\varepsilon}$ above to obtain

$$
\begin{aligned}
J^{\varepsilon}\left(u^{\varepsilon}, u_{t}^{\varepsilon}\right) & \leq \frac{1}{2}\left\|\left(u^{\varepsilon}, u_{t}^{\varepsilon}\right)\right\|_{X_{\varepsilon}^{1 / 2} \times X_{\varepsilon}^{0}}^{2}+\frac{b}{2}\left\|u^{\varepsilon}\right\|_{L^{2}(\Omega)}\left\|u_{t}^{\varepsilon}\right\|_{L^{2}(\Omega)}-\int_{\Omega} F\left(u^{\varepsilon}\right) \\
& \leq \frac{3}{4}\left\|\left(u^{\varepsilon}, u_{t}^{\varepsilon}\right)\right\|_{X_{\varepsilon}^{1 / 2} \times X_{\varepsilon}^{0}}^{2}+C \int_{\Omega}\left(\left|u^{\varepsilon}\right|^{p+1}+\left|u^{\varepsilon}\right|^{2}+\left|u^{\varepsilon}\right|\right) \\
& \leq C\left\|\left(u^{\varepsilon}, u_{t}^{\varepsilon}\right)\right\|_{X_{\varepsilon}^{1 / 2} \times X_{\varepsilon}^{0}}^{2}+C \quad \forall t \geq 0 .
\end{aligned}
$$

This will follow easily after an application of Hölder's inequality, an adequent Young's inequality, and the Nirenberg-Gagliardo's inequality $[1,8]$

$$
\|u\|_{L^{p+1}(\Omega)} \leq C\|u\|_{H^{1}(\Omega)}^{\alpha}\|u\|_{L^{2}(\Omega)}^{(1-\alpha)} \quad \text { for } \alpha=\frac{N}{2}-\frac{N}{p+1} .
$$

In fact, since $p<1+2 / N$, this implies $\alpha(p+1)<1$. Hence raising both sides of the above inequality (3.28) to the power $p+1$ and using the Young inequality $a b \leq(1 / s) a^{s}+\left(1 / s^{\prime}\right) b^{s^{\prime}}$ with $a, b \geq 0, s=2 / \alpha(p+1)>1$ such that $1 / s+1 / s^{\prime}=1$, we estimate the first term of the second sum in (3.26). But since this is not as immediate because

$$
s^{\prime} \frac{(1-\alpha)(p+1)}{2} \neq 1
$$

we have to choose some $\vartheta>1$ such that

$$
\frac{\alpha(p+1)}{2}+\frac{(1-\alpha)(p+1)}{2 \vartheta}=1, \quad \frac{(1-\alpha)(p+1)}{2 \vartheta} s^{\prime}=1,
$$

which yields $\vartheta=(1-\alpha)(p+1) /(2-\alpha(p+1))$. Thus, after expressing

$$
\int_{\Omega}|u|^{p+1} \leq C\left(\int_{\Omega}|\nabla u|^{2}+\int_{\Omega}|u|^{2}\right)^{\alpha(p+1) / 2}\left(\int_{\Omega}|u|^{2 / \vartheta}\right)^{(1-\alpha)(p+1) \vartheta / 2},
$$

it is possible to apply Young's inequality successively to furnish

$$
\int_{\Omega}|u|^{p+1} \leq 2\left(\int_{\Omega}|\nabla u|^{2}+\int_{\Omega}|u|^{2}\right)+C .
$$


The following term of the sum in (3.26) needs not be estimated, while the last estimates easily as

$$
\int_{\Omega}\left|u^{\varepsilon}\right| \leq|\Omega|^{1 / 2}\left\|u^{\varepsilon}\right\|_{L^{2}(\Omega)} \leq \frac{1}{2}\left\|u^{\varepsilon}\right\|_{L^{2}(\Omega)}^{2}+\frac{|\Omega|}{2}
$$

by virtue of Hölder and Young inequalities. Finally, in the last estimate (3.27), the constant $C \geq 0$ is the maximum of the resulting constants of the computations following from (3.26).

Now, with (3.27) in (3.23), we obtain

$$
\frac{d J^{\varepsilon}\left(u^{\varepsilon}, u_{t}^{\varepsilon}\right)}{d t} \leq-\frac{b C}{8} J^{\varepsilon}\left(u^{\varepsilon}, u_{t}^{\varepsilon}\right)+C .
$$

Consequently, solving this differential inequality, we find for $t \geq 0$ that

$$
J^{\varepsilon}\left(u^{\varepsilon}, u_{t}^{\varepsilon}\right) \leq e^{-(b C / 8) t} J^{\varepsilon}\left(u_{0}^{\varepsilon}, u_{1}^{\varepsilon}\right)+C\left(1-e^{-(b C / 8) t}\right) .
$$

It follows again, by (3.27) and the hypotheses on the initial data, taking (3.25) into account, that

$$
\begin{aligned}
& \limsup _{t \rightarrow \infty}\left\|\left(u^{\varepsilon}, u_{t}^{\varepsilon}\right)\right\|_{X_{\varepsilon}^{1 / 2} \times X_{\varepsilon}^{0}}^{2} \\
& \quad \leq \limsup _{t \rightarrow \infty}\left\{e^{-(b C / 8) t} J^{\varepsilon}\left(u_{0}^{\varepsilon}, u_{1}^{\varepsilon}\right)+C\left(1-e^{-(b C / 8) t}\right)\right\}+C \leq C
\end{aligned}
$$

and the first assertion is proved.

To complete the proof, we observe in (3.18) that when $b=0$ we have the classical Lyapunov functional. Moreover, from the second estimate in (3.19), we readily see for any solution $\left(u^{\varepsilon}, u_{t}^{\varepsilon}\right)$ of $(1.1)$ through $\left(u_{0}^{\varepsilon}, u_{1}^{\varepsilon}\right)$ that

$$
\frac{d J^{\varepsilon}\left(u^{\varepsilon}, u_{t}^{\varepsilon}\right)}{d t}=-\beta \int_{\Omega}\left|u_{t}^{\varepsilon}\right|^{2} \leq 0 \Longrightarrow J^{\varepsilon}\left(u^{\varepsilon}, u_{t}^{\varepsilon}\right) \leq J^{\varepsilon}\left(u_{0}^{\varepsilon}, u_{1}^{\varepsilon}\right)
$$

for all $t \geq 0$. Next, using (3.27), we find

$$
\int_{0}^{\infty}\left\|u_{t}^{\varepsilon}\right\|_{L^{2}(\Omega)}^{2} \leq 2 J^{\varepsilon}\left(u_{0}^{\varepsilon}, u_{1}^{\varepsilon}\right) \leq C \rho+C
$$

and the proof of the theorem is concluded.

Following results in $[5,6,7]$ and references therein, it is easy to deduce the following corollary from the foregoing theorem (Theorem 3.4). 
Corollary 3.5. There exists a global compact attractor $\mathcal{A}^{\varepsilon} \subset X_{\varepsilon}^{1 / 2} \times X_{\varepsilon}^{0}$ of (1.1).

The proof we give below is similar to the one found in [5]. Its advantage lies in that we can obtain compactness of the attractor by a density argument. Moreover, in (1.1) we are considering an even much simpler linear damping term.

Proof. Our argument runs as follows. Consider problem (1.1) for sufficiently regular data $d^{\varepsilon}, b^{\varepsilon} \in C^{1},\left(u_{0}^{\varepsilon}, u_{1}^{\varepsilon}\right) \in \Phi=C_{0}^{\infty}(\Omega)$, and let $u^{\varepsilon}=v^{\varepsilon}+w^{\varepsilon}$ be the unique strong solution such that

$$
\begin{gathered}
v_{t t}^{\varepsilon}+\beta v_{t}^{\varepsilon}+L^{\varepsilon} v^{\varepsilon}=0, \quad v^{\varepsilon}(0)=u_{0}^{\varepsilon}, \quad v_{t}^{\varepsilon}(0)=u_{1}^{\varepsilon}, \\
w_{t t}^{\varepsilon}+\beta w_{t}^{\varepsilon}+L^{\varepsilon} w^{\varepsilon}=f\left(u^{\varepsilon}\right), \quad w^{\varepsilon}(0)=w_{t}^{\varepsilon}(0)=0 .
\end{gathered}
$$

Then, in the setting of Theorem 3.4, if we consider the equation in $w^{\varepsilon}$ and let $p=r / q>1$ for some $r \geq(2 N+4) / N$ fixed, since the inclusions $H^{1}(\Omega) \hookrightarrow L^{s}(\Omega)$ are compact, for $s \geq 1$ satisfying in $N \geq 3$ the condition given below, we have from [9, Chapter I.5] that the imbeddings

$$
L^{\infty}\left(0, T, H^{1}(\Omega)\right) \cap W^{1, \infty}\left(0, T, L^{2}(\Omega)\right) \hookrightarrow L^{m}\left(0, T, L^{s}(\Omega)\right)
$$

are also compact for any $1<m<\infty, 1 \leq s<2 N /(N-2)$. Thus, the nonlinearity

$$
f: L^{r}\left(Q_{T}\right) \mapsto L^{q}\left(Q_{T}\right)
$$

is well defined and compact in $L^{N r /(N+2)}\left(Q_{T}\right)$. Further, it is easy to see from the energy associated that the mapping

$$
L^{N r /(N+2)}\left(Q_{T}\right) \ni f\left(u^{\varepsilon}\right) \longmapsto\left(w^{\varepsilon}, w_{t}^{\varepsilon}\right) \in X_{\varepsilon}^{1 / 2} \times X_{\varepsilon}^{0}
$$

is continuous. On the other hand, using the semigroup $\left(v^{\varepsilon}(t), v_{t}^{\varepsilon}(t)\right)=$ $T^{\varepsilon}(t)\left(v_{0}^{\varepsilon}, v_{1}^{\varepsilon}\right), t \geq 0$, we have

$$
\left\|\left(v^{\varepsilon}, v_{t}^{\varepsilon}\right)\right\|_{X_{\varepsilon}^{1 / 2} \times X_{\varepsilon}^{0}} \leq M e^{-(\beta / 2) t}\left\|\left(v_{0}^{\varepsilon}, v_{1}^{\varepsilon}\right)\right\|_{X_{\varepsilon}^{1 / 2} \times X_{\varepsilon}^{0}}, \quad \forall t \geq 0,
$$

with

$$
\frac{\beta}{2}=\operatorname{Re}\left(\frac{\beta \pm \sqrt{\beta^{2}-\mu_{1}^{\varepsilon}}}{2}\right)
$$


424 Finite-dimensional asymptotic behaviour

where $\mu_{1}^{\varepsilon} \in \sigma\left(L^{\varepsilon}\right)$ is the first eigenvalue to the problem $L^{\varepsilon} \psi=\mu \psi, \psi \in$ $H^{1}(\Omega)$.

Next, set $\mathscr{A}^{\varepsilon}=\cap_{t \geq 0} \overline{T^{\varepsilon}(t) \Phi}$ with $T^{\varepsilon}(t) \Phi \stackrel{\text { def }}{=}\left(u^{\varepsilon}(t), u_{t}^{\varepsilon}(t)\right)$ being the strong solution of (1.1) for regular data $\left(u_{0}^{\varepsilon}, u_{1}^{\varepsilon}\right) \in \boldsymbol{D}$. Then, clearly $\mathcal{A}^{\varepsilon}$ is a closed set in $X_{\varepsilon}^{1 / 2} \times X_{\varepsilon}^{0}$ and, from the above, is compact. Thus, by density we have a global compact attractor $\mathscr{A}^{\varepsilon}=\cap_{t \geq 0} \overline{T^{\varepsilon}(t) \bar{B}}$ in $X_{\varepsilon}^{1 / 2} \times X_{\varepsilon}^{0}$, where $B$ denotes an absorbing set for (1.1), and the proof of the corollary is complete.

\subsection{Large diffusivity limiting problem.}

We will now complete our study of the large diffusivity asymptotic behaviour of the solutions to (1.1).

THEOREM 3.6. Let $\left(u^{\varepsilon}, u_{t}^{\varepsilon}\right)$ denote the solution to (1.1) and let $\left(u_{\Omega}, \dot{u}_{\Omega}\right)$ be such that

$$
\ddot{u}_{\Omega}+\beta \dot{u}_{\Omega}+\left(\frac{|\Gamma|}{|\Omega|} f_{\Gamma} b+\lambda\right) u_{\Omega}=h_{\Omega}(u), \quad\left(u_{\Omega}^{0}, u_{\Omega}^{1}\right) \in \mathbb{R}^{2},
$$

where $h_{\Omega}(u)=f_{\Omega} f(u)$. Assume that the hypotheses in Theorem 3.4 hold and that $u_{1}^{\varepsilon} \rightarrow u_{\Omega}^{1}$ strongly in $L^{2}(\Omega)$ as $\varepsilon \rightarrow 0$. Then for all $t \geq 0$,

$$
\left(u^{\varepsilon}, u_{t}^{\varepsilon}\right) \longrightarrow\left(u_{\Omega}, \dot{u}_{\Omega}\right) \text { strongly in } X_{\varepsilon}^{1 / 2} \times X_{\varepsilon}^{0},
$$

as $\varepsilon \rightarrow 0$. In particular, the family of attractors $\left\{\mathcal{A}^{\varepsilon} \cup \mathcal{A}\right\}_{\varepsilon>0}$, where $\mathcal{A}$ is a global attractor for (3.45), verifies

$$
\lim _{\varepsilon \rightarrow 0} \sup _{\left(u^{\varepsilon}, u_{t}^{\varepsilon}\right) \in \mathcal{A}^{\varepsilon}} \inf _{\left(u_{\Omega}, \dot{u}_{\Omega}\right) \in \mathcal{A}}\left\|\left(u^{\varepsilon}, u_{t}^{\varepsilon}\right)-\left(u_{\Omega}, \dot{u}_{\Omega}\right)\right\|_{X_{\varepsilon}^{1 / 2} \times X_{\varepsilon}^{0}}=0 ;
$$

in other words, it is upper semicontinuous in $\varepsilon=0$.

Proof. Consider the energy identity

$$
\begin{aligned}
& \frac{1}{2}\left\|\left(u^{\varepsilon}, u_{t}^{\varepsilon}\right)\right\|_{X_{\varepsilon}^{1 / 2} \times X_{\varepsilon}^{0}}^{2}+\beta \int_{Q_{T}}\left|u_{t}^{\varepsilon}\right|^{2} \\
& =\int_{Q_{T}} f\left(u^{\varepsilon}\right) u_{t}^{\varepsilon}+\frac{1}{2}\left\|\left(u_{0}^{\varepsilon}, u_{1}^{\varepsilon}\right)\right\|_{X_{\varepsilon}^{1 / 2} \times X_{\varepsilon}^{0}}^{2} .
\end{aligned}
$$


Since $f\left(u^{\varepsilon}\right) \in L^{q}\left(Q_{T}\right)$ and $u_{t}^{\varepsilon} \in L^{\infty}\left(0, T, L^{2}(\Omega)\right) \subset L^{\infty}\left(0, T, L^{q^{\prime}}(\Omega)\right)$, we have, using the Hölder's inequality and Young's inequality (2.7), that

$$
\left|\int_{Q_{T}} f\left(u^{\varepsilon}\right) u_{t}^{\varepsilon}\right| \leq 4 C\left\|f\left(u^{\varepsilon}\right)\right\|_{L^{q}\left(Q_{T}\right)}^{2}+\frac{1}{4}\left\|u_{t}^{\varepsilon}\right\|_{L^{\infty}\left(0, T, L^{2}(\Omega)\right)}^{2} .
$$

Consequently, in (3.48), we obtain

$$
\begin{aligned}
& \|\left.\left(u^{\varepsilon}, u_{t}^{\varepsilon}\right)\right|_{X_{\varepsilon}^{1 / 2} \times X_{\varepsilon}^{0}} ^{2}+\beta \int_{Q_{T}}\left|u_{t}^{\varepsilon}\right|^{2} \\
& \quad \leq C\left(\left\|f\left(u^{\varepsilon}\right)\right\|_{L^{q}\left(Q_{T}\right)}^{2}+\left\|\left(u_{0}^{\varepsilon}, u_{1}^{\varepsilon}\right)\right\|_{X_{\varepsilon}^{1 / 2} \times X_{\varepsilon}^{0}}^{2}\right) .
\end{aligned}
$$

On the other hand, the Nirenberg-Gagliardo's inequality (3.28), with $r \geq(2 N+4) / N$, implies that $f\left(u^{\varepsilon}\right) \in L^{q}\left(Q_{T}\right)$ is bounded in norm for all $\varepsilon>0$ since, using the coercive estimate (1.5), we have $\left\|u^{\varepsilon}\right\|_{X_{\varepsilon}^{1 / 2}}^{2} \rightarrow \infty$ as $\varepsilon \rightarrow 0$. Therefore, from (3.50), we have the convergence including (2.10). Moreover, using (3.40) yields

$$
f\left(u^{\varepsilon}\right) \longrightarrow f(v) \text { strongly in } L^{N r /(N+2)}\left(Q_{T}\right),
$$

as $\varepsilon \rightarrow 0$.

It now suffices to observe that, by the uniform boundedness in norm for $\varepsilon>0$ of the initial data, we have $u_{0}^{\varepsilon} \rightarrow u_{\Omega}^{0}$ strongly in $X_{\varepsilon}^{1 / 2}$, as $\varepsilon \rightarrow$ 0 , and we can apply the second part of Theorem 2.2 to obtain that the limit $(v, \dot{v})=\left(u_{\Omega}, \dot{u}_{\Omega}\right)$ is strong in $Y_{T}$ and verifies (3.45) in the sense of distributions for all $t \geq 0$, which proves (3.46).

We proceed to observe that (3.16) also holds in (3.45). Hence, the finite-dimensional equation has a compact attractor $\mathcal{A} \subset \mathbb{R}^{2}$, and since the above limiting process remains true on the family of attractors $\left\{\mathscr{A}^{\varepsilon} \cup\right.$ $A\}_{\varepsilon}$, we have, using [6, Section 4.10.2, page 165], that the orbits on these attractors satisfy (3.47) and the proof is complete.

Finally, in retrospect, in the given framework to (1.1), it is evident that (3.47) implies the long-time dynamics of the semilinear wave equation (1.1) with large diffusion taking place in all parts of the spatial domain $\Omega$, and it is essentially close to the one described by the second-order ordinary differential equation (3.45).

Although it is clear that under the additional hypothesis on the data in Remark 2.3 we have

$$
\lim _{\varepsilon \rightarrow 0} \int_{\Omega} d^{\varepsilon}(x)\left|\nabla u^{\varepsilon}\right|^{2}=0, \quad \forall t \geq 0,
$$


the regularity of the data to (1.1) does not allow one to obtain a fine exponential decay estimate of the above quantity as in the parabolic case studied in [4]. This quantity (3.52) tells us that in the given model (1.1) spatial homogenization of solutions to a constant function in space occurs sufficiently rapid when (1.4) is experienced. In other words, if we view the energy functional

$$
E^{\varepsilon}(t)=\left\|\left(u^{\varepsilon}, u_{t}^{\varepsilon}\right)\right\|_{X_{\varepsilon}^{1 / 2} \times X_{\varepsilon}^{0}}^{2}+\beta \int_{Q_{T}}\left|u_{t}^{\varepsilon}\right|^{2}
$$

as expressing spatial heterogeneities of the solutions to problem (1.1) (see, e.g., [10]), then (1.4) implies that these are eliminated sufficiently rapid.

\section{Acknowledgment}

The author is very grateful to Professor A. Rodríguez Bernal (U.C.M. Mat. Apl. Spain) for introducing the problem to him and for providing the reference [3]. He is also grateful to the referees for numerous improvements.

\section{References}

[1] A. R. Bernal, Lecture Notes of the Course: Ecuaciones de Evolución Semilinear, 2000, Curso 96-97, U.C.M. MAT. APL., Madrid, Spain.

[2] A. R. Bernal and R. Willie, Linear parabolic problem and large diffusion, submitted to Series B. Jrnl of Discrete and Continuous Dynamical Systems, 2002.

[3] A. N. Carvalho, Spatial homogeneity in damped hyperbolic equations, CDSNSReport Series CDSNS92-75, Georgia Institute of Technology, School of Mathematics, Georgia, 1990.

[4] E. Conway, D. Hoff, and J. Smoller, Large time behavior of solutions of systems of nonlinear reaction-diffusion equations, SIAM J. Appl. Math. 35 (1978), no. 1, $1-16$.

[5] E. Feireisl, Global attractors for semilinear damped wave equations with supercritical exponent, J. Differential Equations 116 (1995), no. 2, 431-447.

[6] J. K. Hale, Asymptotic Behavior of Dissipative Systems, Mathematical Surveys and Monographs, vol. 25, American Mathematical Society, Rhode Island, 1988.

[7] J. K. Hale and G. Raugel, Upper semicontinuity of the attractor for a singularly perturbed hyperbolic equation, J. Differential Equations 73 (1988), no. 2, 197214.

[8] D. Henry, Geometric Theory of Semilinear Parabolic Equations, Lecture Notes in Mathematics, vol. 840, Springer-Verlag, Berlin, 1981.

[9] J.-L. Lions, Quelques Méthodes de Résolution des Problèmes aux Limites Non Linéaires, Dunod, Gauthier-Villars, Paris, 1969 (French). 
[10] J. D. Murray, Mathematical Biology, Biomathematics, vol. 19, Springer-Verlag, Berlin, 1993.

[11] M. Renardy and R. C. Rogers, An Introduction to Partial Differential Equations, Texts in Applied Mathematics, vol. 13, Springer-Verlag, New York, 1993.

[12] R. Willie, Ecuaciones de reaccion-difusion con difusion alta, Ph.D. thesis, Universidad Complutense de Madrid, Madrid, 2000.

Robert Willie: Departamento de Matemática Aplicada, Facultad de Ciencias Matemáticas, Universidad Complutense de Madrid, 28040 Madrid, Spain

Current address: Department of Mathematics, University of Zimbabwe, M.P. 167, Harare, Zimbabwe

E-mail addresses: rwillie@sunma4.mat.ucm.es; rwillie@math.ac.uz.zw 


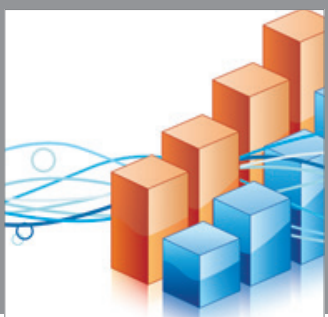

Advances in

Operations Research

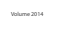



\section{The Scientific} World Journal


International Journal of

Mathematics and

Mathematical

Sciences
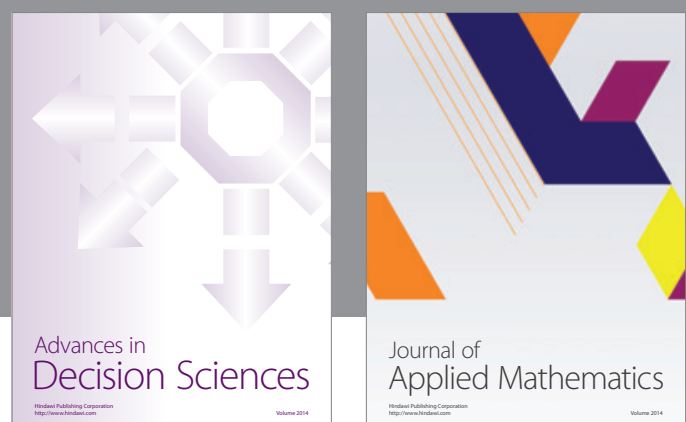

Journal of

Applied Mathematics
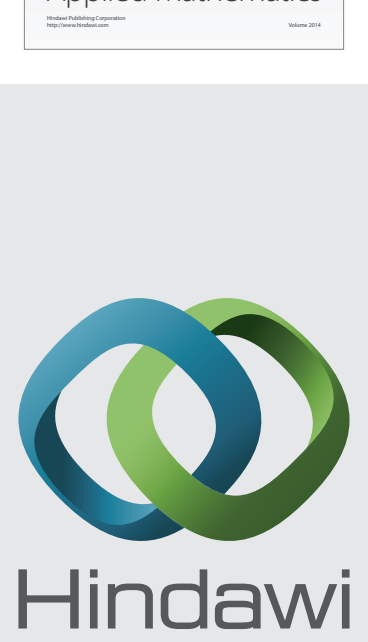

Submit your manuscripts at http://www.hindawi.com


Mathematical Problems in Engineering
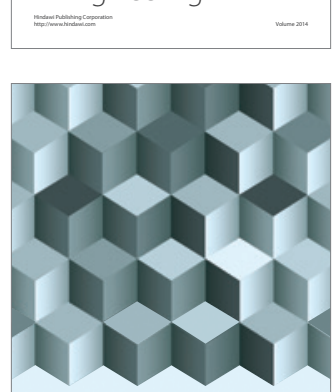

Journal of

Function Spaces
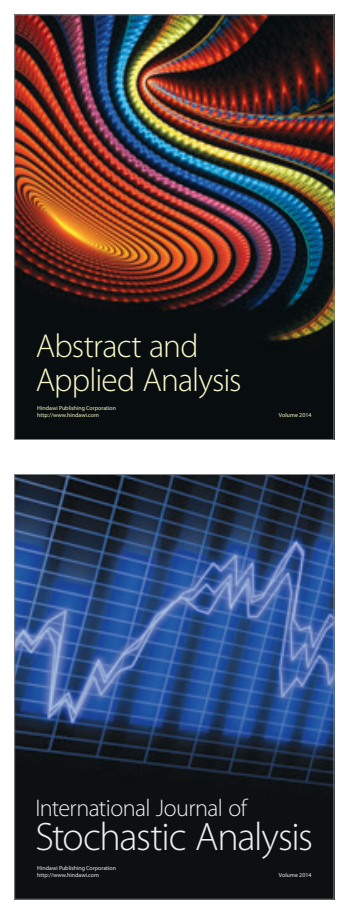

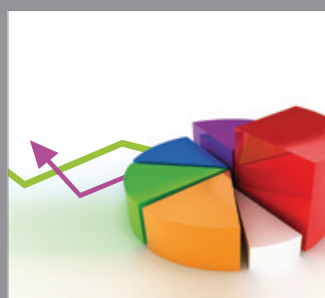

ournal of

Probability and Statistics

Promensencen
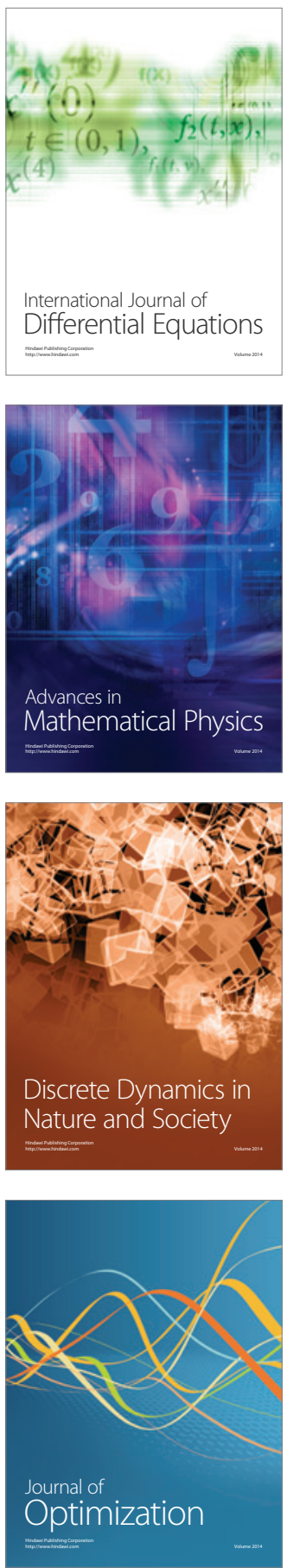